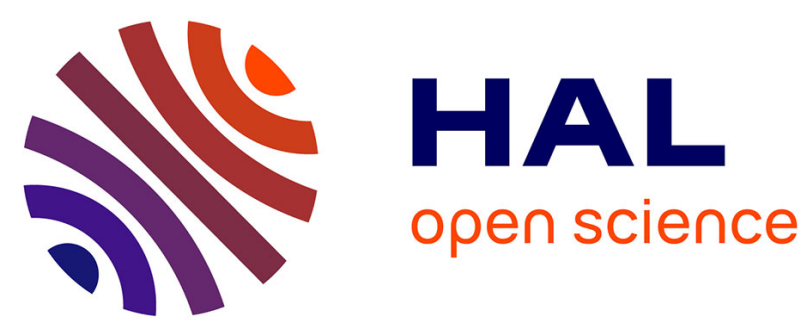

\title{
An Experimental Investigation of Discretized Homogeneous Differentiators: Pneumatic Actuator Case
}

Loïc Michel, Subiksha Selvarajan, Malek Ghanes, Franck Plestan, Yannick Aoustin, Jean-Pierre Barbot

\section{- To cite this version:}

Loïc Michel, Subiksha Selvarajan, Malek Ghanes, Franck Plestan, Yannick Aoustin, et al.. An Experimental Investigation of Discretized Homogeneous Differentiators: Pneumatic Actuator Case. IEEE Journal of Emerging and Selected Topics in Industrial Electronics, 2021, IEEE Journal of Emerging and Selected Topics in Industrial Electronics, 2 (3), pp.227-236. 10.1109/JESTIE.2021.3061924 . hal-03414142

\author{
HAL Id: hal-03414142 \\ https://hal.science/hal-03414142
}

Submitted on 4 Nov 2021

HAL is a multi-disciplinary open access archive for the deposit and dissemination of scientific research documents, whether they are published or not. The documents may come from teaching and research institutions in France or abroad, or from public or private research centers.
L'archive ouverte pluridisciplinaire HAL, est destinée au dépôt et à la diffusion de documents scientifiques de niveau recherche, publiés ou non, émanant des établissements d'enseignement et de recherche français ou étrangers, des laboratoires publics ou privés. 


\title{
An experimental investigation of discretized homogeneous differentiators: pneumatic actuator case
}

\author{
Loïc Michel (D), Subiksha Selvarajan (D), Malek Ghanes (D), Member, IEEE, Franck Plestan (D), Member, IEEE, \\ Yannick Aoustin (D), and Jean-Pierre Barbot (D), Senior Member, IEEE
}

\begin{abstract}
It is well known that the homogeneous differentiator strategies reduce the chattering in the implementation of sliding mode algorithms and the recent implicit framework based projector provides very interesting properties when associated to homogeneous design. In this paper, it is proposed to evaluate different strategies of homogeneous differentiators based on Euler explicit and promising Euler semi-implicit strategies, for which discrete implementations have been realized on a pneumatic actuator. Experimental results highlight the benefit of a Euler semi-implicit based structure using a projector.
\end{abstract}

Index Terms-Differentiator, sampled data, pneumatic actuator.

\section{INTRODUCTION}

Real-time signal differentiation can be performed using sliding modes techniques [1], for which interesting robustness properties are highlighted despite the sensibility to measurement noise. Some classes of differentiators, including homogeneous, high order sliding mode, high-gain and ALIEN differentiators (e.g. [2], [3]) are discussed and compared in [4], [5] in the framework of indoor small blimp robot's altitude control, but without taking into account the influence of the sampling period. In the last decade, Acary \& Brogliato [6] introduced the implicit discretization, whose purpose is to replace the sign function by an implicit projector. This technique would overcome some limitations of the classical sliding-mode control such as a strong reduction of the chattering effect as well as robustness of the control under lower sampling frequencies and preservation of the global stability. Recent investigations (see [7]-[10]) have shown very promising results and experimental validations of some implicit based sliding mode control algorithms have been successfully performed (e.g. [11], [12]). Homogeneity is a very interesting powerful approach. For example, if a local stability is obtained due to the dilation, this framework allows extending this local property to global settings (see e.g. [13]), see also controllability property [14] and geometric properties [15]. Regarding the design of differentiators, homogeneity

Loïc Michel, Subiksha Selvarajan, Malek Ghanes and Franck Plestan are with École Centrale de Nantes-LS2N, UMR 6004 CNRS, Nantes, France (e-mail: loic.michel@ec-nantes.fr, subiksha.selvarajan@eleves.ec-nantes.fr, malek.ghanes@ec-nantes.fr, franck.plestan@ec-nantes.fr).

Yannick Aoustin is with Université de Nantes-LS2N, UMR 6004 CNRS, Nantes, France (e-mail: yannick.aoustin@univ-nantes.fr).

Jean-Pierre Barbot is with LS2N, UMR 6004 CNRS, Nantes, and Quartz Laboratory EA 7393, France (e-mail: barbot@ensea.fr)

Manuscript received XX; revised XX. allows to guaranty finite time convergence (see also [16] for nearly fixed time convergence). Moreover, an appropriate choice of homogeneity degrees allows making a good trade-off between uncertain perturbation rejection and less-sensitivity to noise [1]. Considering sliding mode control or differentiator under sampling, besides convergence issues, the so-called implicit projector [6] allows reducing the chattering effects. In case of homogeneous control or differentiator, the semiimplicit discretization strategies based on a mix of implicit and explicit Euler schemes have been recently proposed in the literature (see e.g. [17]-[19]).

The aim of this paper is to provide a comparative study of recent results regarding these semi-implicit based differentiation methodologies. These works provide several ways to implement the correction term vector of the differentiators. The original implicit strategy implemented experimentally in [12] for sliding mode control deals with the implicit projector. After that, regarding the implementation of homogeneous differentiators, some semi-implicit techniques have been proposed: quasi-linearization method [18], method ensuring the solution continuity [17] and homogeneous-based generalization of the projector [19]. More precisely, the homogeneous semi-implicit-based discretization [17] and the semi-implicit homogeneous based pseudo-linearization differentiators [18] are compared to the classical homogeneous explicit methods; this allows to highlight the implementation properties of the homogeneous semi-implicit differentiator based projector in terms of good estimation in presence of noisy measurements and derivative estimation efficiency. This differentiator is inspired by the work in [19]; the discretization of a homogeneous differentiator is difficult due to the existence of some singularities at zero with respect to the evaluation of the second derivative of the Taylor expansion. The evaluation framework of these differentiating methods is conducted on a nonlinear pneumatic actuator where the goal is to obtain the velocity and acceleration estimations from the position measurement of the pneumatic actuator. The physical parameters of this bench are poorly known and difficult to identify with precision. However the laboratory has a great expertise of this experimental bench. Therefore, this bench is ideal for testing differentiators, as it has been already proved for control testing purposes, see [12], [20], [21].

The remainder of the present paper is organized as follows. The considered model to be differentiated is given Section III. In Section III, the existing discretization methods are 
reviewed. The experimental framework is described in Section IV Section V presents the simplified model of the test-bed. The experimental evaluation including some discussion of the results is presented in Section VI Some concluding remarks are given in Section VII.

\section{MODEL UNDER CONSIDERATION}

The goal is to estimate the velocity and the acceleration of the pneumatic actuator exclusively from the measured position, using some real-time differentiation techniques. From this point of view, motivated by the pneumatic actuator application [20], for which the velocity and acceleration are needed for control purposes, monitoring, etc. The continuous model under consideration is the following one:

$$
\Sigma:\left\{\begin{array}{ccc}
\dot{x}_{1} & = & x_{2} \\
\dot{x}_{2} & = & x_{3} \\
\dot{x}_{3} & = & d \\
y & = & x_{1}+\eta
\end{array}\right.
$$

where $x_{1}, x_{2}$, and $x_{3}$ are respectively the position, the velocity and the acceleration; $d$ represents the physical unknown parameters, but also the known system dynamics that are function of the state and control; $y$ is the measure of $x_{1}$ with additional white noise $\eta$.

\section{HOMOGENEOUS FINITE TIME DIFFERENTIATORS UNDER DISCRETIZATION}

\section{A. Notations}

A structure of cascaded differentiators is considered for which a schematic representation is given in Fig. 1 Throughout the paper, the following notations are used: $y^{a}=y$ is the measured output, $z_{1}^{a}, z_{2}^{a}$ are respectively the estimated position and velocity given by the first differentiator, $y^{b}=z_{2}^{b}$ is the output of the second differentiator, $z_{1}^{b}$ and $z_{2}^{b}$ are respectively the velocity and acceleration estimates by the second differentiator and $e_{1}^{a}=y^{a}-z_{1}^{a}=y-z_{1}^{a}$ and $e_{1}^{b}=y^{b}-z_{1}^{b}=z_{2}^{a}-z_{1}^{b}$ are respectively the output errors of the first and second differentiators. The variable $\bullet+$ corresponds to the value at the instant $\bullet((k+1) h)$. Moreover $\lceil\bullet\rfloor^{\gamma}$ is for $|\bullet|{ }^{\gamma} \operatorname{sgn}(\bullet)$.

\section{B. Continuous second order homogeneous differentiator}

In order to obtain the position $x_{1}$, the velocity $x_{2}$ and the acceleration $x_{3}$ of the pneumatic actuator, a cascaded iterative of homogeneous differentiator structure is proposed in order to estimate the velocity $v$ and the acceleration $a$. This gives different choices of homogeneous exponent $\alpha_{1}$ and $\alpha_{2}$ for the differentiators, but with the same structure of second order:

$$
\begin{aligned}
& \dot{z}_{1}^{i}=\lambda_{1}^{i}\left\lceil e_{1}^{i}\right\rfloor^{\alpha_{i}}+z_{2}^{i} \\
& \dot{z}_{2}^{i}=\lambda_{2}^{i}\left\lceil e_{1}^{i}\right\rfloor^{2 \alpha_{i}-1}
\end{aligned}
$$

with $\alpha_{i} \in\left[\frac{1}{2}, 1[\right.$ for $i \in\{a, b\}$.
The position measurement of the pneumatic actuator in the experimental testbed is realized under sampling, with sampling period $h$. From the discretization point of view, $h$ is the constant period of sampling. Then, the implementation of differentiator 22 must be done. This differentiator is not $\mathbb{C}^{1}$, consequently the Taylor expansions of the observation error and the estimated state solution have second derivatives, which are singular at $x=0$ for homogeneous differentiators (2) when $\alpha_{i}<1$. Therefore, only a first order discretization scheme is allowed, but which one? This is the framework of the end of this section.

\section{Some ways of discretization}

The differentiators for $i \in\{a, b\}$ include a naturally decreasing homogeneity degree according to the order of the differentiation. To allow adjusting the homogeneity degree according to the order of the estimated state, and inspired by the technique developed in [22]-[24], a nonlinear differentiator is designed as a cascaded association of second order differentiators. In other words, a recursive reduced order differentiator is composed of a first differentiator, denoted "a", that estimates $z_{1}^{a}$ and $z_{2}^{a}$ from $y^{a}=y$ and that feeds the second differentiator, denoted " $\mathrm{b}$ ", that estimates $z_{1}^{b}$ and $z_{2}^{b}$ from $y^{b}=z_{2}^{a}$ (see Fig. 1 .

1) Explicit Euler discretization (E2D)

The most popular discretization is the Explicit Euler discretization, which gives respectively for (2):

$$
\begin{aligned}
& z_{1}^{i+}=z_{1}^{i}+h\left(\lambda_{1}^{i}\left\lceil e_{1}^{i}\right\rfloor^{\alpha_{i}}+z_{2}^{i}\right) \\
& z_{2}^{i+}=z_{2}^{i}+h\left(\lambda_{2}^{i}\left\lceil e_{1}^{i}\right\rfloor^{2 \alpha_{i}-1}\right)
\end{aligned}
$$

As it is pointed out by a lot of articles, this solution is not the most appropriate method for discretizing differential inclusions or simply to preserve the stability under discretization see [10].

2) Semi-Implicit Discretization based on Explicit Sign function (SIDES)

It is one of the reasons why in [6], an implicit Euler discretization scheme was proposed in the framework of sliding mode control. After that in [17], a semi-implicit Euler scheme was proposed in order to preserve the solution continuity and the good property of the implicit method with respect to the stability. This gives for (2) the following differentiator form:

$$
\begin{aligned}
& z_{1}^{i+}=z_{1}^{i}+h\left(\lambda_{1}^{i}\left|e_{1}^{i+}\right|^{\alpha_{i}} \operatorname{sgn}\left(y_{1}^{i}-z_{1}^{i}\right)+z_{2}^{i+}\right) \\
& z_{2}^{i+}=z_{2}^{i}+h\left(\lambda_{2}^{i}\left|e_{1}^{i+}\right|^{2 \alpha_{i}-1} \operatorname{sgn}\left(y_{1}^{i}-z_{1}^{i}\right)\right)
\end{aligned}
$$

Remark that (4) can be solved analytically for $\alpha_{i} \in$ $\{0.5,1,2\}$, then for $\alpha_{i}=0.5$, the equation:

$$
e_{1}^{i+}+h \lambda_{1}^{i}\left|e_{1}^{i+}\right|^{0.5} \operatorname{sgn}\left(e_{1}^{i}\right)-e_{1}^{i}=0
$$

is solved including $w_{i}=\left|e_{1}^{i+}\right|^{0.5}$. From (5), the two following cases are considered: 
- Considering $e_{1}^{i}<0$, and choosing $e_{1}^{i+}<0$, one obtain:

$$
e_{1}^{i+}=-\left(\frac{-h \lambda_{1}^{i}+\sqrt{\left(h \lambda_{1}^{i}\right)^{2}+4\left|e_{1}^{i}\right|}}{2}\right)^{2}
$$

- Considering $e_{1}^{i}>0$, and choosing $e_{1}^{i+}>0$, one obtain:

$$
e_{1}^{i+}=\left(\frac{-h \lambda_{1}^{i}+\sqrt{\left(h \lambda_{1}^{i}\right)^{2}+4\left|e_{1}^{i}\right|}}{2}\right)^{2}
$$

and we summarize both solutions as:

$$
e_{1}^{i+}=\left(\frac{-h \lambda_{1}^{i}+\sqrt{\left(h \lambda_{1}^{i}\right)^{2}+4\left|e_{1}^{i}\right|}}{2}\right)^{2} \operatorname{sgn}\left(e_{1}^{i}\right)
$$

From this, (4) with $\alpha_{i}=0.5$ becomes:

$$
\begin{aligned}
& z_{1}^{i+}=z_{1}^{i}+h \lambda_{1}^{i} w_{i} \operatorname{sgn}\left(e_{1}^{i}\right)+h z_{2}^{i+} \\
& z_{2}^{i+}=z_{2}^{i}+h \lambda_{2}^{i} \operatorname{sgn}\left(e_{1}^{i}\right)
\end{aligned}
$$

where

$$
w_{i}=\frac{-h \lambda+\sqrt{(h \lambda)^{2}+4\left|e_{1}^{i}\right|}}{2} .
$$

3) Semi-Implicit Discretization based on pseudo Linearisation (SIDL)

In [18], a pseudo linearization of (2) and semi-implicit scheme were proposed, thus replacing $\operatorname{sgn}\left(e_{1}\right)$ by $\frac{e_{1}}{\left|e_{1}\right|}$. Moreover, in the appendix of [18], it was shown that for $x_{1} \rightarrow 0$ (in the article $\sigma_{1} \rightarrow 0$ ), the two eigenvalues of the pseudo-inverse matrix are considered equal to zero by continuity argument, even if the solution is not defined in $x=0$. Indeed, both eigenvalue limits in $x=0^{+}$and $x=0^{-}$are equal to $0^{-}$. It reads:

$$
\begin{aligned}
& \dot{z}_{1}^{i}=\lambda_{1}^{i}\left|e_{1}^{i}\right|^{\alpha_{i}-1} e_{1}^{i}+z_{2}^{i} \\
& \dot{z}_{2}^{i}=\lambda_{2}^{i}\left|e_{1}^{i}\right|^{2\left(\alpha_{i}-1\right)} e_{1}^{i}
\end{aligned}
$$

Also, a semi-implicit discretization of the pseudo linearization (SIDL) is proposed:

$$
\begin{aligned}
& z_{1}^{i+}=z_{1}^{i}+h\left(\lambda_{1}^{i}\left|e_{1}^{i}\right|^{\alpha_{i}-1} e_{1}^{i+}+z_{2}^{i+}\right) \\
& z_{2}^{i+}=z_{2}^{i}+h\left(\lambda_{2}^{i}\left|e_{1}^{i}\right|^{2\left(\alpha_{i}-1\right)} e_{1}^{i+}\right)
\end{aligned}
$$

Note that in (11), only the linear terms are assigned at time $k+1$. To solve these equations, the first line of $[11$ is modified as follows:

$$
y_{1}^{i+}-z_{1}^{i+}=y_{1}^{i+}-z_{1}^{i}-h\left(\lambda_{1}^{i}\left|e_{1}^{i}\right|^{\alpha_{i}-1} e_{1}^{i+}-z_{2}^{i+}\right)
$$

thus (11) can be rewritten:

$$
\begin{aligned}
\left(\begin{array}{c}
e_{1}^{i+} \\
z_{2}^{i+}
\end{array}\right)= & \left(\begin{array}{c}
y_{1}^{i+}-z_{1}^{i} \\
z_{2}^{i}
\end{array}\right)+ \\
& h\left(\begin{array}{cc}
-\lambda_{1}^{i}\left|e_{1}^{i}\right|_{\alpha_{i}-1} & 1 \\
\lambda_{2}^{i}\left|e_{1}^{i}\right|^{2\left(\alpha_{i}-1\right)} & 0
\end{array}\right)\left(\begin{array}{c}
e_{1}^{i+} \\
z_{2}^{i+}
\end{array}\right)
\end{aligned}
$$

and this gives:

$$
\left(\begin{array}{c}
e_{1}^{i+} \\
z_{2}^{i+}
\end{array}\right)=\left(\begin{array}{cc}
1+h \lambda_{1}^{i}\left|e_{1}^{i}\right|^{\alpha_{i}-1} & h \\
-h \lambda_{2}^{i}\left|e_{1}^{i}\right|^{2\left(\alpha_{i}-1\right)} & 1
\end{array}\right)^{-1}\left(\begin{array}{c}
y_{1}^{i+}-z_{1}^{i} \\
z_{2}^{i}
\end{array}\right)
$$

Then explicit solution of (11) reads:

$$
\begin{gathered}
\left(\begin{array}{c}
z_{1}^{i+} \\
z_{2}^{i+}
\end{array}\right)=\left(\begin{array}{c}
y_{1}^{i+} \\
0
\end{array}\right)+\left(\begin{array}{cc}
-1 & 0 \\
0 & 1
\end{array}\right) \times \\
\left(\begin{array}{cc}
1+h \lambda_{1}^{i}\left|e_{1}^{i}\right|^{\alpha_{i}-1} & h \\
-h \lambda_{2}^{i}\left|e_{1}^{i}\right|^{2\left(\alpha_{i}-1\right)} & 1
\end{array}\right)^{-1}\left(\begin{array}{c}
y_{1}^{i+}-z_{1}^{i} \\
z_{2}^{i}
\end{array}\right)
\end{gathered}
$$

It is important to remark that some computational problems can occur when $e_{1}^{i}$ is very close to zero.

4) Semi-Implicit Discretization based on Implicit Sign function with Projector (SIDP)

There exists another way to semi-implicitly discretize the cascade of second order differentiators:

$$
\begin{aligned}
& z_{1}^{i+}=z_{1}^{i}+h\left(z_{2}^{i+}+\lambda_{1}^{i}\left|e_{1}^{i}\right|^{\alpha_{i}} \mathcal{N}\left(e_{1}^{i}, \alpha_{i}, \lambda_{1}^{i}\right)\right) \\
& z_{2}^{i+}=z_{2}^{i}+h\left(\lambda_{2}^{i}\left|e_{1}^{i}\right|^{2 \alpha_{i}-1} \mathcal{N}\left(e_{1}^{i}, \alpha_{i}, \lambda_{1}^{i}\right)\right)
\end{aligned}
$$

The projector $\mathcal{N}\left(e_{1}^{i}, \alpha_{i}, \lambda_{1}^{i}\right)$ is defined as follows:

$$
\mathcal{N}\left(e_{1}^{i}, \alpha_{i}, \lambda_{1}^{i}\right):=\left\{\begin{array}{l}
\frac{\left\lceil e_{1}^{i}\right\rfloor^{1-\alpha_{i}}}{\lambda_{1}^{i} h}, \text { if }\left|e_{1}^{i}\right|^{1-\alpha_{i}}<\lambda_{1}^{i} h \\
\operatorname{sgn}\left(e_{1}^{i}\right), \text { if }\left|e_{1}^{i}\right|^{1-\alpha_{i}} \geq \lambda_{1}^{i} h
\end{array}\right.
$$

where $i \in\{a, b\}$. As it is previously noted in the case of a cascade of two second order differentiators of the form (13), the homogeneity degree can thus be adjusted independently according to how much the output is noisy. This is not possible with (9) where $\alpha_{i}$ is fixed to 0.5 .

5) Semi-Implicit Discretization based on implicit Sign function with Modified Projector (SIDMP)

Let us remark that in order to improve the robustness with respect to the noise, a modification could be provided for the projector as follows: the projector $\mathcal{N}\left(e_{1}^{i}, \alpha_{i}, \lambda_{1}^{i}\right)$ can be replaced in 13 by the following one $\mathcal{N}_{\theta}\left(e_{1}^{i}, \alpha_{i}, \lambda_{1}^{i}\right):=$

$$
\left\{\begin{array}{l}
\frac{(1-\theta)\left\lceil e_{1}^{i}\right\rfloor^{1-\alpha_{i}}}{\lambda_{1}^{i} h}, \text { if }(1-\theta)\left|e_{1}^{i}\right|^{1-\alpha_{i}}<\lambda_{1}^{i} h \\
\operatorname{sgn}\left(e_{1}^{i}\right), \text { if }\left|e_{1}^{i}\right|^{1-\alpha_{i}} \geq \lambda_{1}^{i} h
\end{array}\right.
$$

with $\theta \in[0, \quad 1[$.

$\theta$ is introduced in order to mitigate the influence of noise on the sliding surface. Roughly speaking, on the sliding surface, for $\theta$ equals to zero, the convergence is a finite time convergence and when $\theta$ is different from zero the convergence is only asymptotic but the differentiator is less sensitive to noise. This is due to the fact that the influence of noise on the sliding surface is multiplied by $(1-\theta)$, thus this improves the behavior of the differentiator in regard to the measurement noise. For that reason, $\mathcal{N}_{\theta}$ is computed with respect to $\theta e_{1}^{i}=e_{1}^{i}-h \lambda_{1}^{i}\left|e_{1}^{i}\right|^{\alpha_{i}} \mathcal{N}_{\theta}\left(e_{1}^{i}, \alpha_{i}, \lambda_{1}^{i}\right)$ instead of $0=e_{1}^{i}-h \lambda_{1}^{i}\left|e_{1}^{i}\right|^{\alpha_{i}} \mathcal{N}\left(e_{1}^{i}, \alpha_{i}, \lambda_{1}^{i}\right)$ for $\mathcal{N}\left(e_{1}^{i}, \alpha_{i}, \lambda_{1}^{i}\right)$.

The schematic representation of the cascaded differentiators, corresponding to (3) is shown in Fig. 1. The term $u$ is the control input applied to the actuator from the controller, $y_{\text {meas }}$ is the measured position from the system (the only available measurement), and the differentiators "a" and "b" represent 
the cascaded nature of the two second order differentiators, giving the estimates of velocity $z_{2}^{a}$ (or $z_{1}^{b}$ ) and acceleration $z_{2}^{b}$, respectively. Two different sampling rates at $h_{1}=0.2 \mathrm{~ms}$ and $h_{2}=0.2 \mathrm{~s}$ are applied.
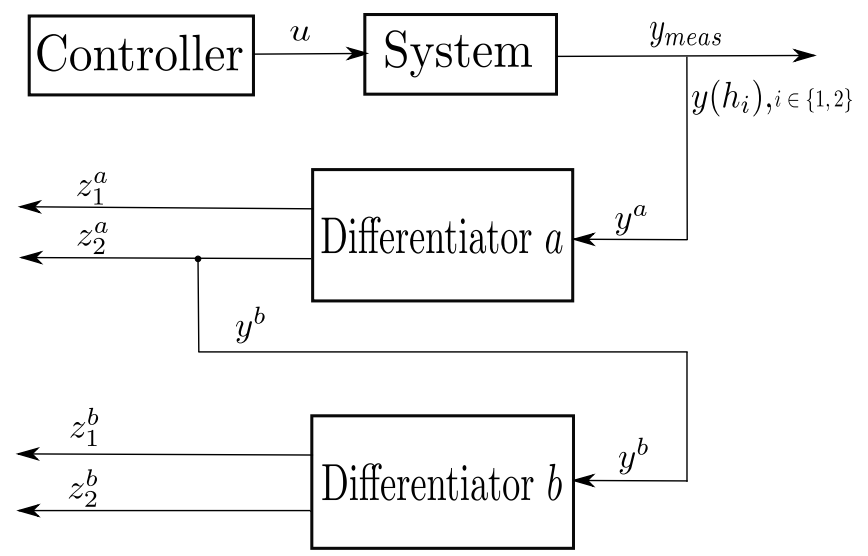

Fig. 1: Synoptic view of the proposed cascaded differentiators to estimate velocity and acceleration.

\section{EXPERIMENTAL TEST-BED}

The pneumatic actuator used to evaluate the differentiators consists of two actuators (or pneumatic pistons) which are controlled by two servo-distributors (see Fig. 2). Each actuator is composed by two chambers denoted by $P$ (positive) and $N$ (negative). The position of one of these two actuators, named "Main actuator" can be controlled, whereas the second actuator, named "Perturbation actuator" and mechanically connected to the "Main" one, allows producing an external perturbation force. Under a nominal 7-bar source pressure, the maximum produced force is $2720 \mathrm{~N}$; furthermore, both actuators have the same physical features: piston diameter of the pneumatic actuator is $80 \mathrm{~mm}$ and rod diameter $25 \mathrm{~mm}$.
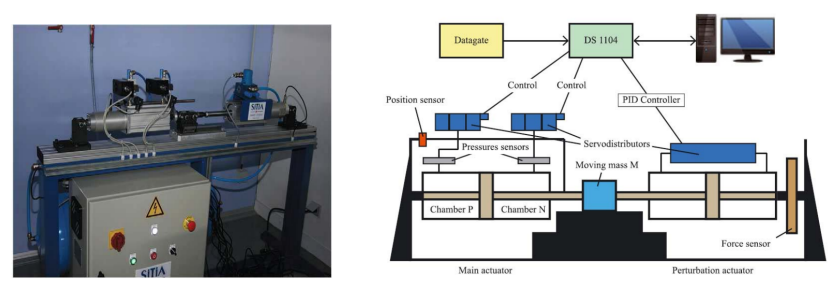

Fig. 2: Left: photography of the pneumatic setup. Right: scheme of the control architecture of the pneumatic setup (details in [20]).

A dSPACE(C) system allows controlling the "Main" actuator as well as monitoring the variables in real-time. Only the position is measured and the "Main" actuator is controlled using a scheme based on both linear and sliding mode approaches (see [25] [21]).

The system itself is nonlinear and is influenced by continuous perturbations generated by the perturbation actuator in Fig. 2 ; thereby, the focus is made on the differentiators to overcome simultaneously this perturbation along with the measurement noise. In this work, a simplified model of the experimental testbed is considered, where a lot of physical parameters are not known (temperature, humidity, operation time, etc). Moreover the measurement noise has to be considered. That is why, homogeneous differentiators strategies are adopted in this work with exponent terms. The latter are fixed to suitable values, which are deduced from experimental tests. These values allow to have a good compromise between sensibility to noise and accuracy / robustness of the estimation error.

\section{Simplified Model of the Test-Bed}

The phenomena which define the physical behavior of this experimental test-bed are complex. They are described through a model based on nonlinear and non-stationary equations including not well-defined parameters. This model has to be simplified by stating hypotheses to simulate the process quickly and with efficiency in order to predict easily its behavior and to define an efficient control as follows [20]:

- air is perfect gas and its kinetic inconsequential;

- pressure and temperature are homogeneous in each chamber;

- mass flow is pseudo-stationary;

- the thermal exchange is only by conduction;

- leakage between system and atmosphere is neglected;

- leakages between the two chambers and between the servodistributor and the jack are negligible;

- temperature variations in each chamber are inconsequential with respect to the supply temperature: $T_{P}=T_{N}=T$;

- process is polytropic and characterized by a coefficient $k$;

- Coulomb friction forces are neglected;

- supply and exhaust pressures are supposed constant;

- effects of the dynamic behavior of the servodistributor are neglected;

- static behavior of servodistributor depends on pressures and control value;

- only the position of the pneumatic actuator is controlled, which means that the problem is a single input-single output (SISO) system, which implies that $u_{P}=-u_{N}=u$; the variable $u$ is the control input of the system.

Taking into account these hypotheses, the mathematical model of the pneumatic actuator (see [20]) reads as:

$$
\begin{aligned}
& \dot{p}_{P}=\frac{k r T}{V_{P}(y)}\left[\varphi_{P}\left(p_{p}\right)+\psi_{P}\left(p_{P}, \operatorname{sgn}(u)\right) u-\frac{S}{r T} p_{P} v\right] \\
& \dot{p}_{N}=\frac{k r T}{V_{N}(y)}\left[\varphi_{N}\left(p_{N}\right)-\psi_{N}\left(p_{N}, \operatorname{sgn}(-u)\right) u+\frac{S}{r T} p_{N} v\right] \\
& \dot{v}=\frac{1}{N}\left[S\left(p_{P}-p_{N}\right)-b_{v} v-F_{\text {ext }}\right] . \\
& \dot{y}=v .
\end{aligned}
$$

with $y$ being the pneumatic actuator position, $v$ its linear velocity, $F_{\text {ext }}$ the external perturbation produced by the perturbation actuator, $p_{P}$ and $p_{N}$ the pressures in chambers $P$ and $N$, respectively, $r$ the perfect gas constant, $b_{v}$ a coefficient 
representing the viscous friction of the process. The measured signal of the mathematical model of the differentiator is assumed to be the output of a two-dimensional integrator with an unknown input. As the cylinder is the physical system, the fundamental principle of dynamics ensures that the perturbations are only located on the acceleration dynamic. Consequently, the perturbations are matched for control purposes and verify the matching observability condition (see [26]) according to the considered measured signal.

\section{EXPERIMENTAL RESULTS}

The control law is implemented on a DS1104 Board (as also used in [27] where a slosh testing rig is driven using the same DSPACE board) and the following working hypothesis are made regarding the controlled trajectory and the choice of the sampling periods.

The control of the position is performed considering linear filter-based estimations of velocity and acceleration with very fast sampling period. Then in the closed-loop, the estimations could be considered as the real states. In this paper, a fast sampling $h_{1}=0.2 \mathrm{~ms}$ and a slow sampling $h_{2}=0.2 \mathrm{~s}$ cases are considered. Remark that all the results provided by the differentiators under study are not used in the control feedback. It is for this reason that the tracking error is not of interest, rather the estimation errors are pertinent and would be discussed henceforth for the comparison of differentiator performances.

\section{a) Assumptions:}

- position reference The desired position reference is $y_{\text {ref }}=$ $0.04 \sin (0.15 t)$ and the control ensures a good tracking of the position (see. Fig. 3);

- velocity reference The estimated velocity $z_{2}$ is compared to the derivative $\dot{y}_{\text {ref }}$;

- acceleration reference The estimated acceleration $z_{3}$ is compared to the second derivative $\ddot{y}_{\text {ref }}$;

- the sampling time of the differentiation can be changed to observe the effects of a possible under-sampling on the resulting differentiated signals: the sampling time is either set to $0.2 \mathrm{~ms}$ or $0.2 \mathrm{~s}$.

The gains for each differentiator are set as follows:

TABLE I: Gain Settings

\begin{tabular}{|l|c|c|c|c|c|c|}
\hline Differentiators & $\lambda_{1}^{a}$ & $\lambda_{2}^{a}$ & $\lambda_{1}^{b}$ & $\lambda_{2}^{b}$ & $\alpha_{1}$ & $\alpha_{2}$ \\
\hline E2D, SID-L/P/MP & 1.5 & 0.625 & 1.5 & 0.625 & 0.75 & 0.7 \\
\hline SIDES & 0.25 & 0.025 & 0.25 & 0.025 & 0.5 & 0.5 \\
\hline
\end{tabular}

The gains of the method SIDES are different from the ones for the other methods. This is due to the fact that for SIDES method $\alpha_{1}=\alpha_{2}=0.5$ and with this value of exponent the differentiators are more sensitive to noise, then it is necessary to tune differently the gain, in order to obtain acceptable results. Also, the SIDMP method includes a parameter $\theta=\frac{1}{2}$. where $e_{v}$ and $e_{a}$ are respectively the estimation errors for the velocity and the acceleration. The Sum of Square Error index (SSE) is given by $\operatorname{SSE}(\bullet)=\frac{1}{n} \sum_{l=1}^{n} \bullet \bullet_{l}^{2}$ with $n$ the file size. For $h_{1}=0.2 \mathrm{~ms}$ and for $h_{2}=0.2 \mathrm{~s}, n$ is respectively set to 100000 and 100.
TABLE II: SSE Indices

\begin{tabular}{|c|c|c|c|c|}
\hline Methods & $\begin{array}{c}\text { SSE }\left(e_{v}\right) \\
h=0.2 \mathrm{~ms}\end{array}$ & $\begin{array}{c}\text { SSE }\left(e_{a}\right) \\
h=0.2 \mathrm{~ms}\end{array}$ & $\begin{array}{c}\text { SSE }\left(e_{v}\right) \\
h=0.2 \mathrm{~s}\end{array}$ & $\begin{array}{c}\text { SSE }\left(e_{a}\right) \\
h=0.2 \mathrm{~s}\end{array}$ \\
\hline E2D & $2.2410^{-6}$ & $5.0410^{-6}$ & $1.7010^{-5}$ & $4.4310^{-5}$ \\
\hline SIDES & $1.6810^{-6}$ & $6.7410^{-6}$ & $1.1810^{-5}$ & $3.1910^{-5}$ \\
\hline SIDL & $6.8910^{-6}$ & $1.1510^{-5}$ & $3.9010^{-6}$ & $1.0310^{-5}$ \\
\hline SIDP & $2.2410^{-6}$ & $5.0410^{-6}$ & $1.6410^{-6}$ & $9.5210^{-6}$ \\
\hline SIDMP & $2.2410^{-6}$ & $5.0410^{-6}$ & $4.3010^{-7}$ & $2.3610^{-6}$ \\
\hline
\end{tabular}

The parameters $\lambda_{1}$ and $\lambda_{2}$ are chosen as follows: $\lambda_{1}=$ $\lambda_{1}^{\prime} \mu^{0.5}$ and $\lambda_{2}=\lambda_{2}^{\prime} \mu$ as classically, where $\lambda_{1}^{\prime}$ and $\lambda_{2}^{\prime}$ ensure a pole-placement and $\mu$ is chosen greater than the maximum of the perturbation. The gains were first tuned for the explicit differentiator to obtain an effective estimation; the same gains were used with all other differentiators, except SIDES (which has the same exponents $\alpha_{1}=\alpha_{2}=0.5$, that implies a retuning of the gains to maintain acceptable performances). This choice of gains is made to compare their performances on an equal footing. Tuning the differentiators with their respective optimal gains would pledge for a fair comparison, but here, in this case, just an equal comparison using equal gains, is presented.

It can be seen from Table II that for $h_{1}=0.2 \mathrm{~ms}$, the SSE in case of SIDP and SIDMP have same values because the projector $\mathcal{N}\left(e_{1}^{i}, \alpha_{i}, \lambda_{1}^{i}\right)$ and $\mathcal{N}_{\theta}\left(e_{1}^{i}, \alpha_{i}, \lambda_{1}^{i}\right)$ are not in effect ${ }^{1}$ and switches between -1 and +1 , and consequently give the same SSE as the E2D method. When the sampling increases $\left(h_{2}=0.2 \mathrm{~s}\right)$, the projector is in effect, thus explaining the differences in the SSE values of SIDP and SIDMP methods.

The position of the actuator is controlled by an explicit sliding mode control law and the corresponding measured position is recorded with a sampling period of $h_{1}=0.2 \mathrm{~ms}$. The signal is then down-sampled to have a sampling period of $h_{2}=0.2 \mathrm{~s}$ as shown in Fig. 3. It could be seen that the sampling at $h_{1}=0.2 \mathrm{~ms}$ is very fast and hence, it appears to be much closer to a continuous signal, whereas at $h_{2}=0.2 \mathrm{~s}$, the signal would have, comparatively, less information which challenges the performances of the differentiators. In such way, the measured position signal (with two different sampling rates) serves as the input for the differentiators under study. From the controlled position being presented in Fig. 3 it could be convincing that the other states (velocity and acceleration) also converge to their corresponding references (with similar accuracy) and hence, the estimations of velocity and acceleration would be compared with $\dot{y}_{r e f}$ and $\ddot{y}_{r e f}$ respectively, in all the plots hereafter.

To go deeper into the performance analysis, the differentiation methods E2D, SIDES, SIDL, SIDP and SIDMP are implemented on the electro-pneumatic actuator system. To avoid confusions, the Sum of Square Error (SSE), including the estimation errors of velocity and acceleration, are computed and are presented in Table II The SSE indices for each method are displayed for easy comparison in Figs. 4 and 5 . It could be concluded from the comparison that the four methods estimate the states with negligible variations at $h_{1}=0.2 \mathrm{~ms}$, but in the case of $h_{2}=0.2 \mathrm{~s}$, SIDMP presents better estimations.

\footnotetext{
${ }^{1}$ The condition $\left|e_{1}^{i}\right|^{\alpha}<\lambda_{1}^{i} h_{1}$ is practically never verified due to the fact that $h_{1}$ is too small.
} 


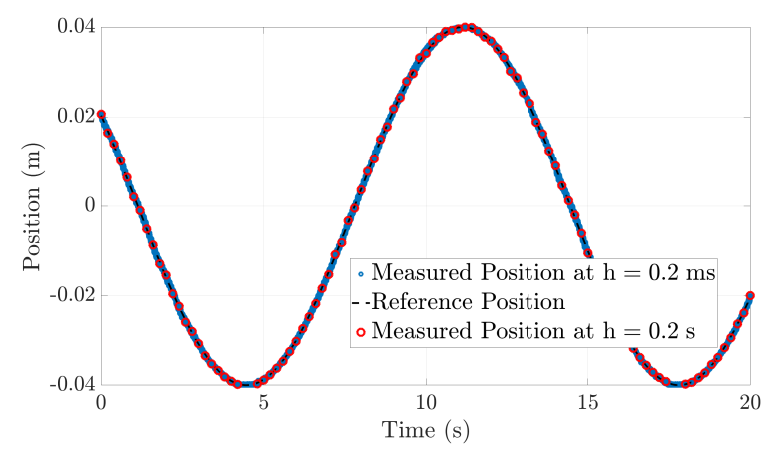

Fig. 3: Reference and Measured Position.

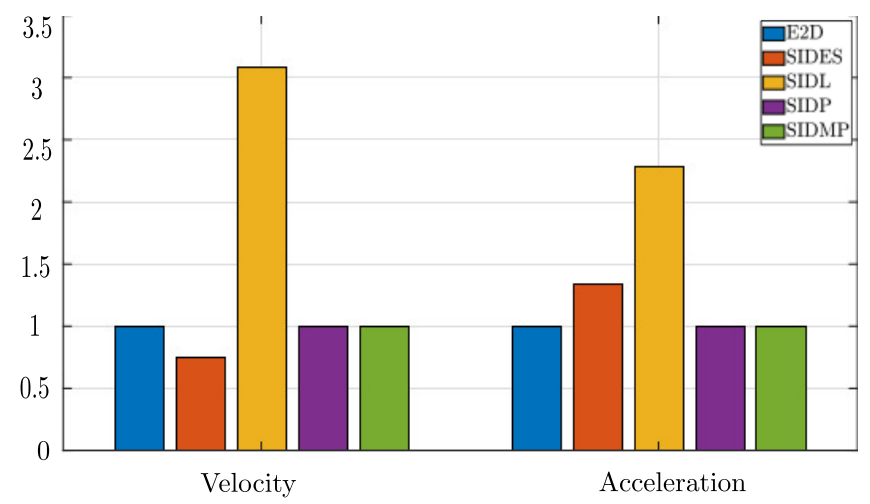

Fig. 4: Normalized SSE for $h_{1}=0.2 \mathrm{~ms}$.

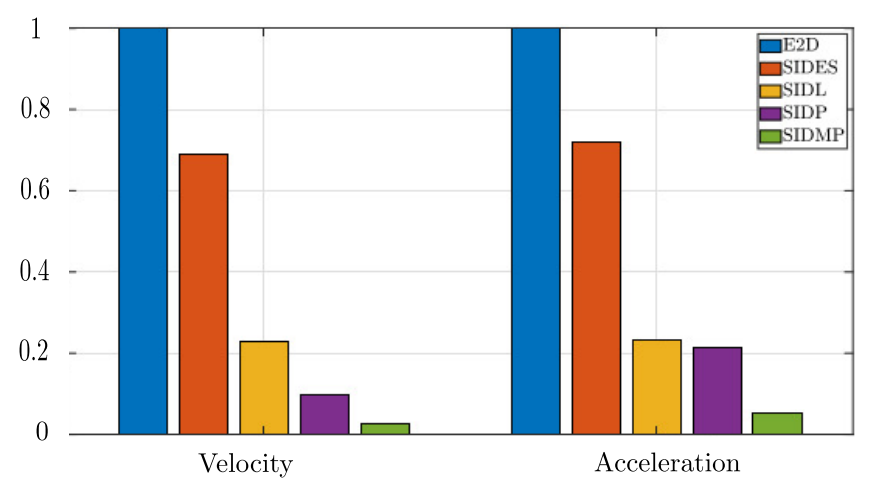

Fig. 5: Normalized SSE for $h_{2}=0.2 \mathrm{~s}$.

Remark that for $h_{1}=0.2 \mathrm{~ms}$, the velocity SSE (resp. acceleration SSE) are normalized with respect to the values of the explicit method $2.2410^{-6}$ (resp. $5.0410^{-6}$ ). Similarly, for $h_{2}=0.2 \mathrm{~s}$, the values of the explicit method are $1.7010^{-5}$ (resp. $4.431^{-5}$ ). According to the Table III. the SSE for $h_{2}=0.2 \mathrm{~s}$ is approximately ten times greater than for $h_{1}=0.2 \mathrm{~ms}$.

Figures 6 and 7 show that the performances of the E2D differentiators are greatly influenced by the sampling period; in the sequel, the results of the E2D differentiators are used for comparison purposes with the other differentiators.

Concerning SIDL, it is observed that the SSE seems pretty bad for $h_{1}=0.2 \mathrm{~ms}$; this is probably due to the fact that the sign function is replaced by $\frac{e_{1}}{\left|e_{1}\right|}$. From our point of view, this method is sensitive to noise. Nevertheless, for $h_{2}=0.2 \mathrm{~s}$, its SSE is greatly improved with respect to E2D method. In the case of $h_{2}=0.2 \mathrm{~s}$, the discretization effect seems to be more influential than the measurement noise. SIDL method works well in regards of discretization effect.

Concerning SIDES, for $h_{2}=0.2 \mathrm{~s}$, the SSE is better than E2D. Nevertheless, the performance seems to be less efficient than for the projector-based methods SIDP and SIDMP. From our point of view, this is due to the fact that this semi-implicit strategy "freezes" the sign function $\left(\operatorname{sgn}\left(e_{k}\right)\right)$.

Concerning SIDMP, the SSE performance is better than for SIDP because this strategy relaxes the constraints of finite time convergence. Doing so, the cascaded differentiator is less sensitive to noise but gives only an asymptotic convergence.

Finally, in order to highlight the analysis given by the SSE indices, in Fig. 9, all acceleration estimations are presented.

For all methods, acceleration behavior is oscillatory. This is due to the fact that mechanical frictions and perturbations on the cylinder are not well compensated by the control and note that the estimated acceleration is compared to the acceleration reference. Moreover, for $h_{2}=0.2 \mathrm{~s}$, some physical information are lost at higher frequencies.

Regarding the case $h_{1}=0.2 \mathrm{~ms}$, all the methods show similar results. This highlights the fact that discretization problems appear for slow sampling. For $h_{2}=0.2 \mathrm{~s}$, the E2D in Fig. 7 seems to be the worst method and the SIDMP is the best one. The SIDES method has a behavior close to the explicit one but with less random behavior. The SIDL has a better behavior than the explicit solutions but the estimation is influenced by noise and also has a phase-shift. The SIDP has a behavior close to SIDMP but it is more sensitive to noise.

In the case of $h_{1}$ (fast sampling period), the performances of semi-implicit and explicit methods are similar. However, it is clearly observed that the implicit performance supersedes the explicit one for larger sampling periods $h_{2}$. Nevertheless, the semi-implicit method is always better for control purposes because the explicit method introduces a bang-bang control input [10], [12].

\section{CONCLUSION}

This work was dedicated to provide an experimental evaluation of recent promising homogeneous differentiators based semi-implicit Euler discretization within the framework of a nonlinear pneumatic actuator. Four differentiators were tested and compared, the homogeneous semi-implicit-based sliding [17], the semi-implicit homogeneous based pseudolinearization [18], and the semi-implicit homogeneous based projector [19] as well. Nevertheless for $h_{1}$ (fast sampling), all the methods are equivalent. This highlights that the discretization problem is for slow sampling. For slow sampling $h_{2}$, it appears clearly that the method SIDMP has the best result (within the focus of comparison).

The classical homogeneous explicit sliding differentiator was also tested and served as a reference for comparisons. Criterion evaluation is made according to the velocity and acceleration estimation performances in presence of measurement noise thanks to a pneumatic setup for which the measured 


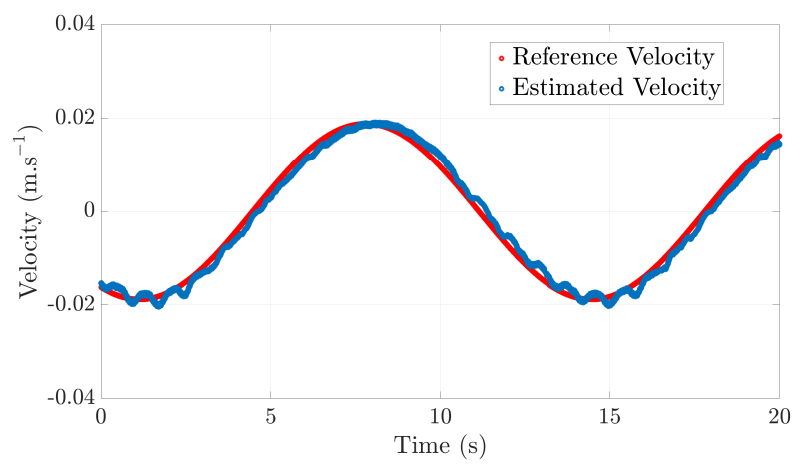

(a) $h=0.2 \mathrm{~ms}$

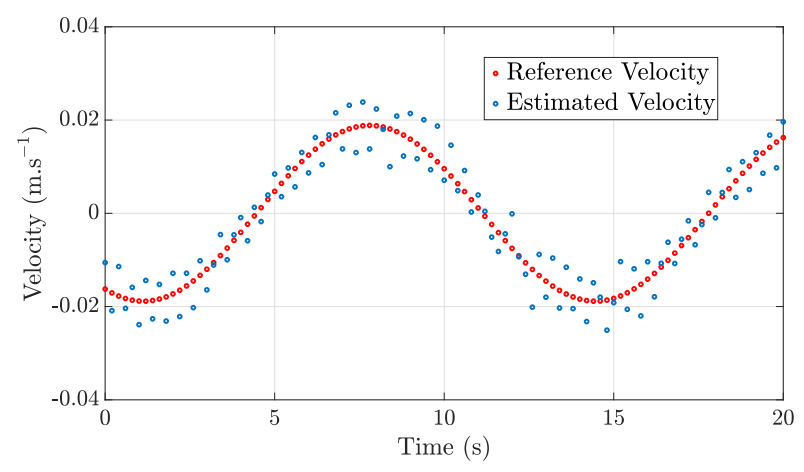

(b) $h=0.2 \mathrm{~s}$

Fig. 6: Estimated Velocity with Explicit Differentiator E2D.

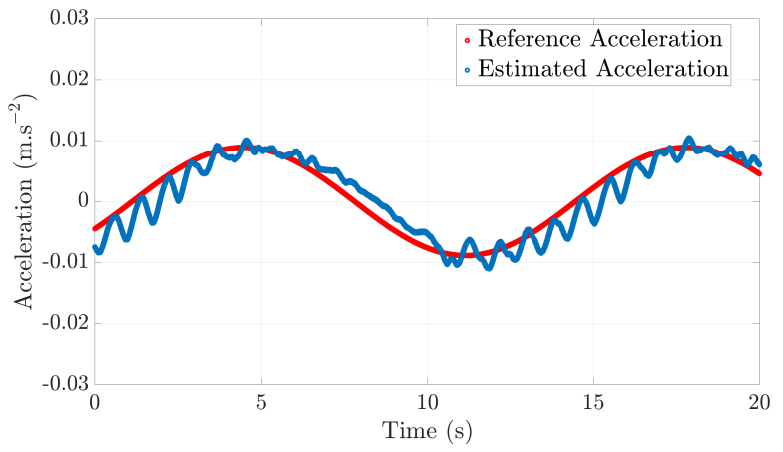

(a) $h=0.2 \mathrm{~ms}$

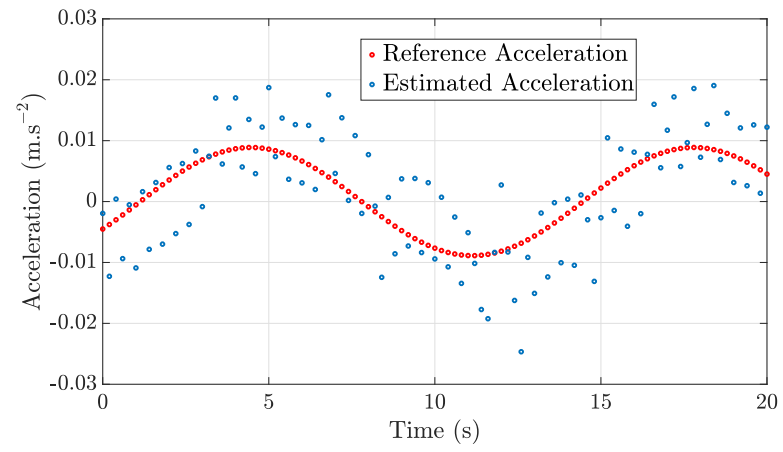

(b) $h=0.2 \mathrm{~s}$

Fig. 7: Estimated Acceleration with Explicit Differentiator E2D.

position has been differentiated to obtain the velocity (first differentiator) and the latter is used as an "output" for the second differentiator in order to obtain the acceleration (cascaded differentiators). As a result, the homogeneous semi-implicit differentiator based projector highlights better performances in terms of the estimation precision and noise rejection.

The proposed methodology will be extended in on-going work to variable exponent differentiators that could deal with noise with an algebraic adaptive law [1].

\section{ACKNOWLEDGMENT}

This work is supported by the ANR project DigitSlid ANR18-CE40-0008-01.

\section{REFERENCES}

[1] M. Ghanes, J. P. Barbot, L. Fridman, A. Levant, and R. Boisliveau, "A new varying-gain-exponent-based differentiator/observer: An efficient balance between linear and sliding-mode algorithms," IEEE Trans. on Automatic Control, vol. 65, no. 12, pp. 5407-5414, 2020.

[2] M. Fliess and H. Sira-Ramírez, Closed-loop Parametric Identification for Continuous-time Linear Systems via New Algebraic Techniques. London: Springer London, 2008, pp. 363-391.

[3] M. Mboup, C. Join, and M. Fliess, "Numerical differentiation with annihilators in noisy environment," Numerical Algorithms, vol. 50, no. 4, pp. 439-467, 2009.

[4] Y. Wang, G. Zheng, D. Efimov, and W. Perruquetti, "Differentiator application in altitude control for an indoor blimp robot," Int. J. of Control, vol. 91, no. 9, pp. 2121-2130, 2018.
[5] M. Mboup, C. Join, M. Fliess, Y. Wang, G. Zheng, D. Efimov, and W. Perruquetti, "Comments on "differentiator application in altitude control for an indoor blimp robot'," Int. J. of Control, vol. 93, no. 5, pp. 1218-1219, 2020.

[6] V. Acary and B. Brogliato, "Implicit Euler numerical scheme and chattering-free implementation of sliding mode systems," Systems \& Control Letters, vol. 59, no. 5, pp. 284 - 293, 2010.

[7] O. Huber, V. Acary, and B. Brogliato, "Comparison between explicit and implicit discrete-time implementations of sliding-mode controllers," in 52nd IEEE Conf. on Decision and Control, 2013, pp. 2870-2875.

[8] B. Brogliato and A. Polyakov, "Globally stable implicit Euler timediscretization of a nonlinear single-input sliding-mode control system," in 2015 54th IEEE Conf. on Decision and Control, 2015, pp. 5426-5431.

[9] V. Acary, B. Brogliato, and Y. V. Orlov, "Chattering-free digital slidingmode control with state observer and disturbance rejection," IEEE Trans. on Automatic Control, vol. 57, no. 5, pp. 1087-1101, 2012.

[10] O. Huber, V. Acary, and B. Brogliato, "Lyapunov stability and performance analysis of the implicit discrete sliding mode control," IEEE Trans. on Automatic Control, vol. 61, no. 10, pp. 3016-3030, 2016.

[11] _ _ "Enhanced matching perturbation attenuation with discrete-time implementations of sliding-mode controllers," in 2014 European Control Conference, 2014, pp. 2606-2611.

[12] B. Wang, B. Brogliato, V. Acary, A. Boubakir, and F. Plestan, "Experimental comparisons between implicit and explicit implementations of discrete-time sliding mode controllers: Toward input and output chattering suppression," IEEE Trans. on Control Systems Technology, vol. 23, no. 5, pp. 2071-2075, 2015.

[13] L. Rosier, "Homogeneous lyapunov function for homogeneous continuous vector field," Systems \& Control Letters, vol. 19, no. 6, pp. 467-473, 1992.

[14] H. Hermes, "Nilpotent and high-order approximations of vector field systems," SIAM Review, vol. 33, no. 2, pp. 238-264, 1991. [Online]. Available: https://doi.org/10.1137/1033050 


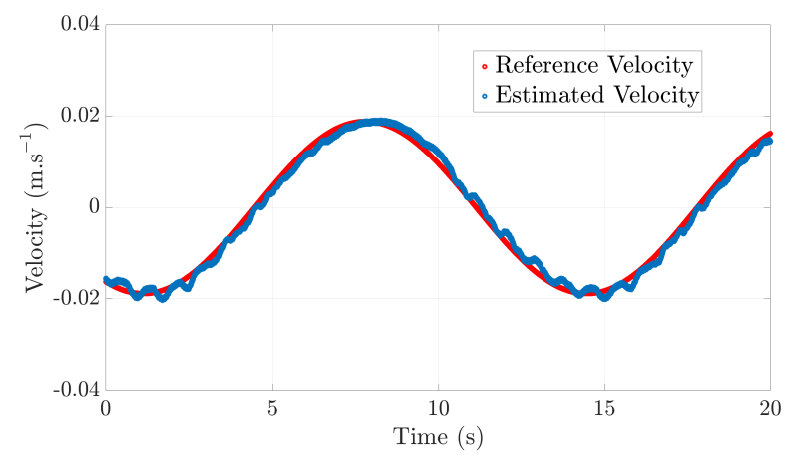

(a) SIDES at $h=0.2 \mathrm{~ms}$

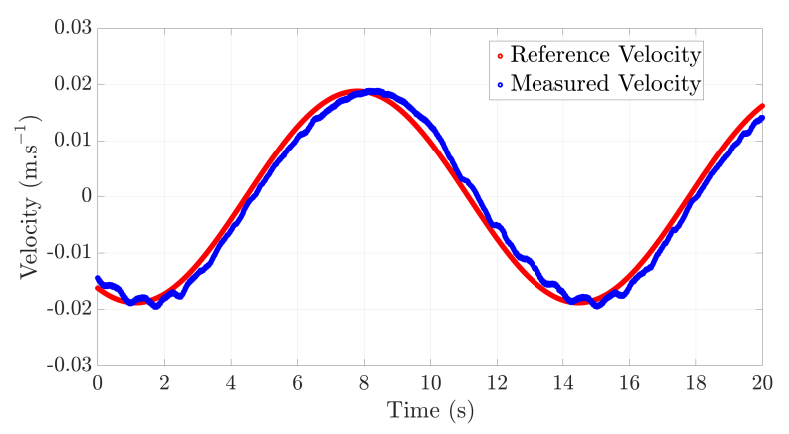

(c) SIDL at $h=0.2 \mathrm{~ms}$

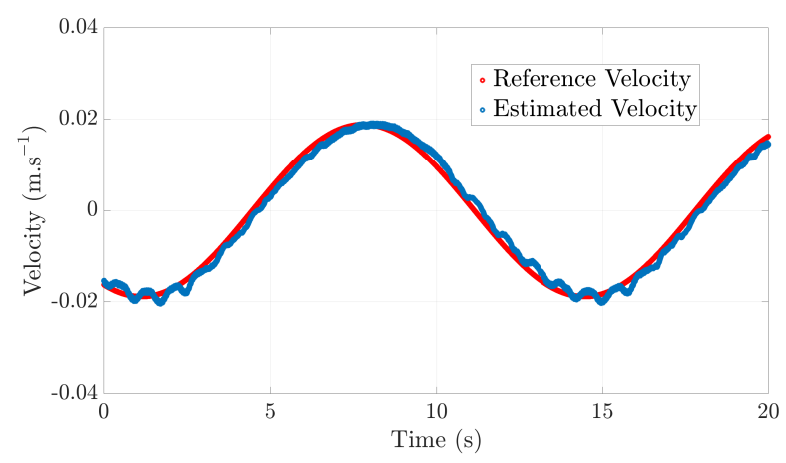

(e) SIDP at $h=0.2 \mathrm{~ms}$

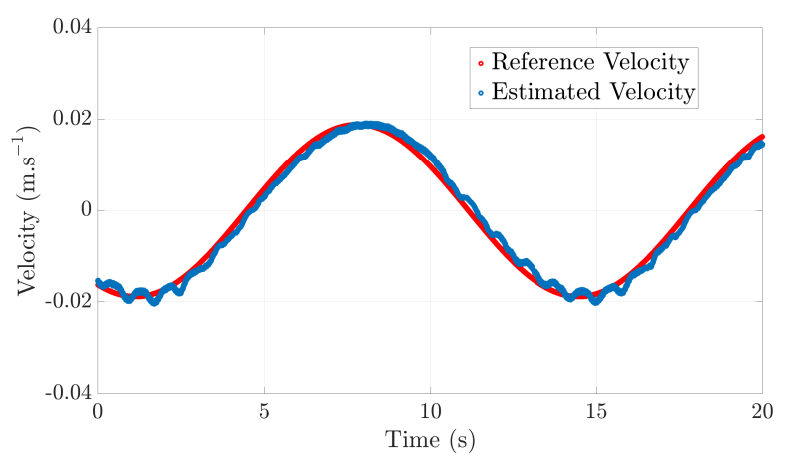

(g) SIDMP at $h=0.2 \mathrm{~ms}$

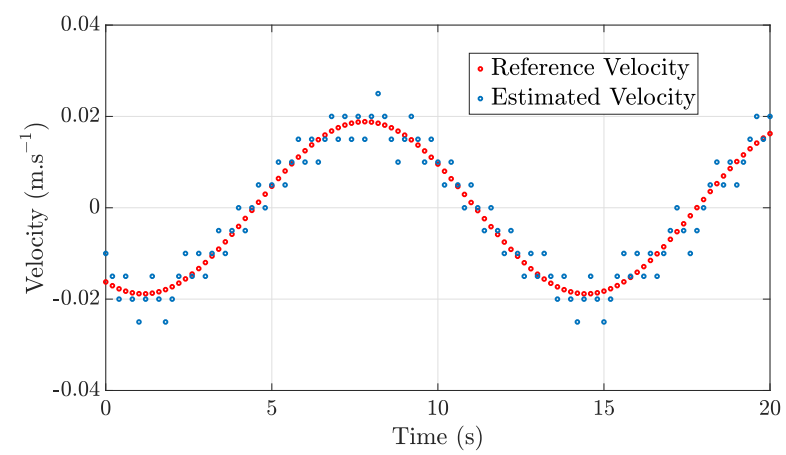

(b) SIDES at $h=0.2 \mathrm{~s}$

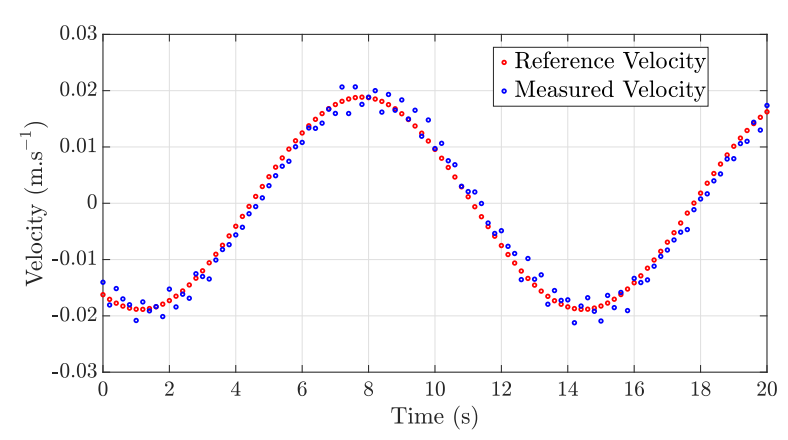

(d) SIDL at $h=0.2 \mathrm{~s}$

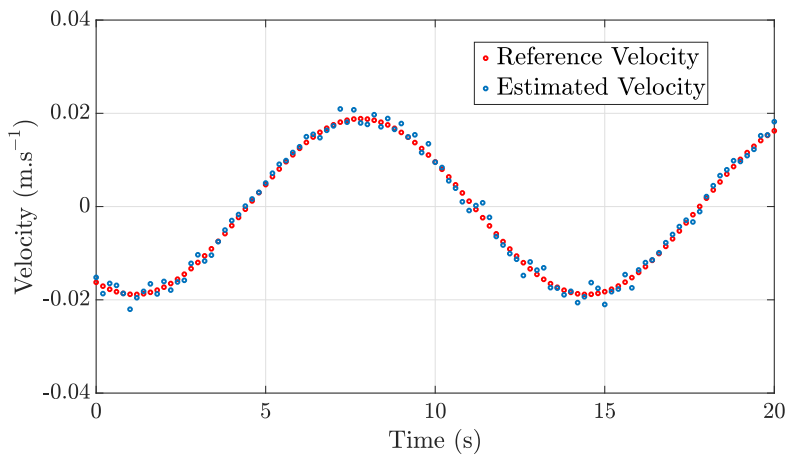

(f) $\mathbf{S I D P}$ at $h=0.2 \mathrm{~s}$

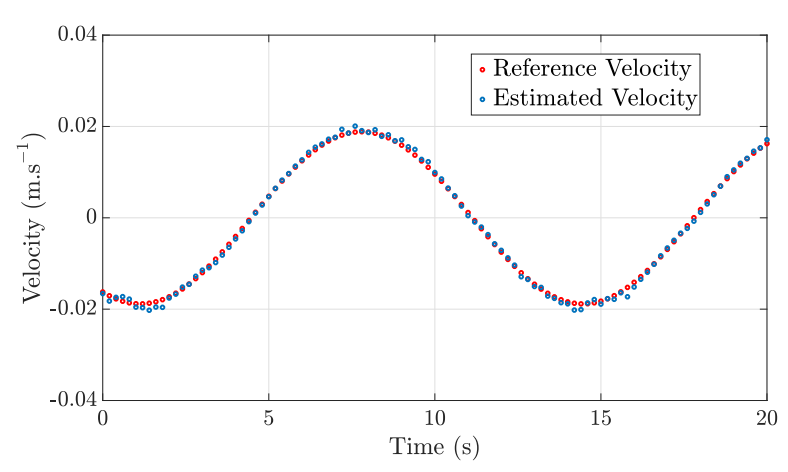

(h) SIDMP at $h=0.2 \mathrm{~s}$

Fig. 8: Velocity Estimates.

[15] M. Kawski, "Geometric homogeneity and stabilization," IFAC Proceedings Volumes, vol. 28, no. 14, pp. 147 - 152, 1995, 3rd IFAC Symposium on Nonlinear Control Systems Design 1995,
Tahoe City, CA, USA, 25-28 June 1995. [Online]. Available: http://www.sciencedirect.com/science/article/pii/S1474667017468224

[16] A. Polyakov, Analysis of Homogeneous Dynamical Systems. Cham: 


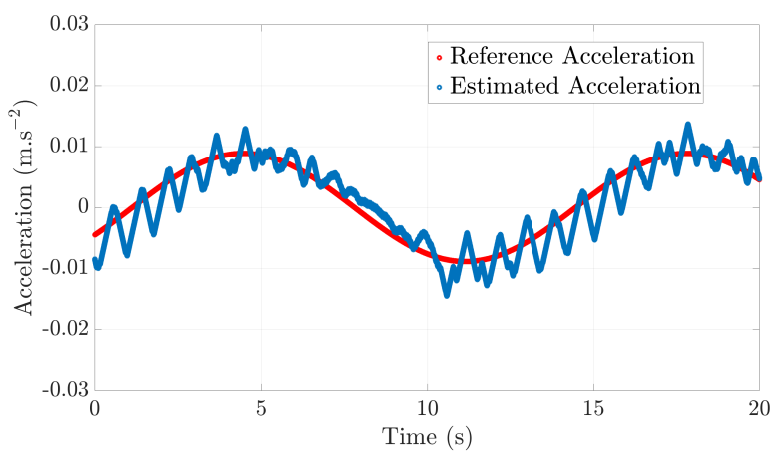

(a) SIDES at $h=0.2 \mathrm{~ms}$

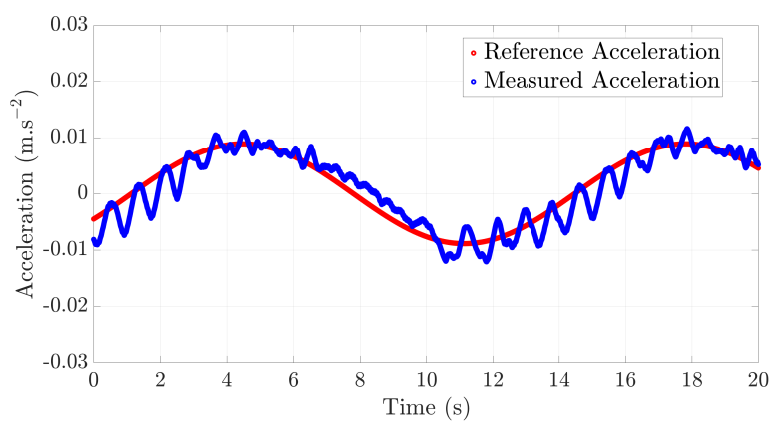

(c) SIDL at $h=0.2 \mathrm{~ms}$

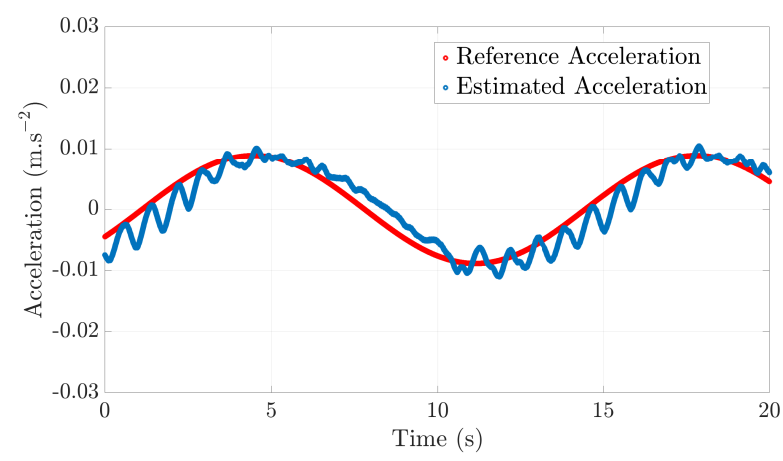

(e) SIDP at $h=0.2 \mathrm{~ms}$

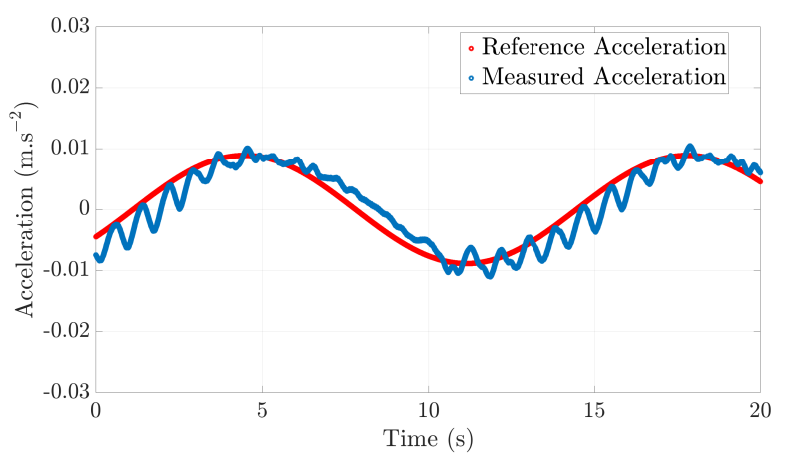

(g) SIDMP at $h=0.2 \mathrm{~ms}$

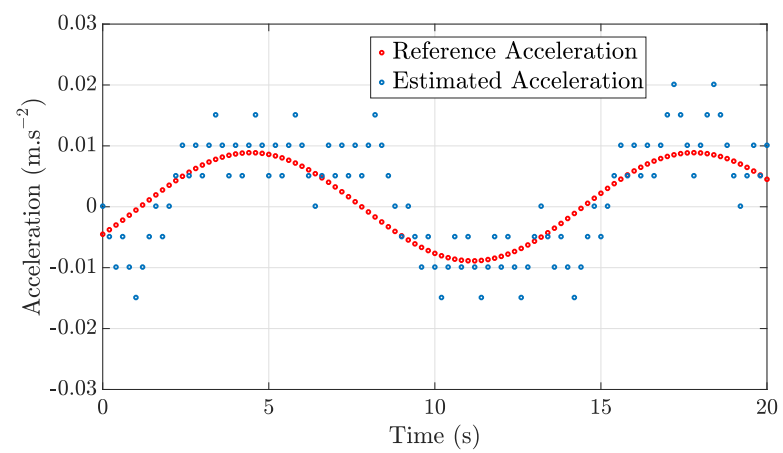

(b) SIDES at $h=0.2 \mathrm{~s}$

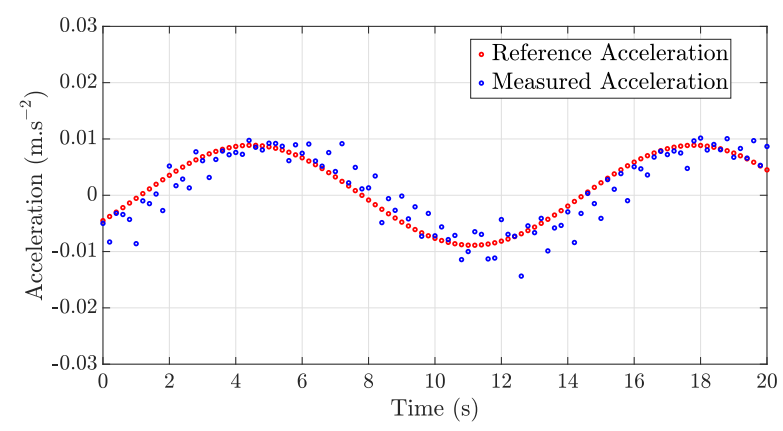

(d) SIDL at $h=0.2 \mathrm{~s}$

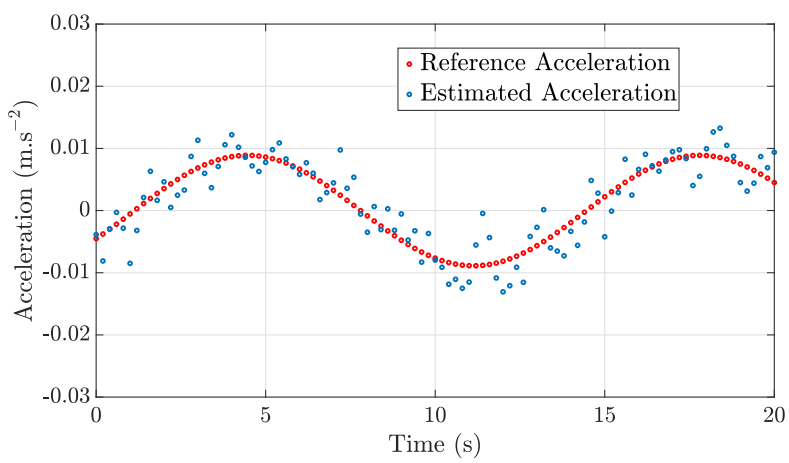

(f) SIDP at $h=0.2 \mathrm{~s}$

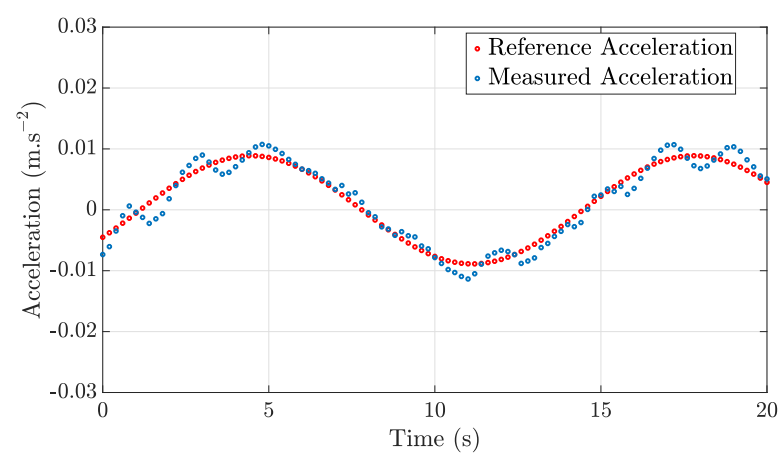

(h) SIDMP at $h=0.2 \mathrm{~s}$

Fig. 9: Acceleration Estimates.

Springer International Publishing, 2020, pp. 225-270. [Online]. Available: https://doi.org/10.1007/978-3-030-38449-4_8

[17] A. Polyakov, D. Efimov, and W. Perruquetti, "Homogeneous differentia- tor design using implicit lyapunov function method," European Control Conference, IEEE, IFAC, pp. -293 288, 2014.

[18] M. Wetzlinger, M. Reichhartinger, M. Horn, L. Fridman, and J. A. 
Moreno, "Semi-implicit discretization of the uniform robust exact differentiator," in 2019 IEEE 58th Conference on Decision and Control, 2019, pp. 5995-6000.

[19] L. Michel, M. Ghanes, F. Plestan, Y. Aoustin, and J. Barbot, "Semiimplicit Euler discretization for homogeneous observer-based control: one dimensional case," in Proc. of the IFAC-V 2020, World Congress, Berlin, Germany, July 2020.

[20] A. Girin and F. Plestan, "A new experimental test bench for a high performance double electropneumatic actuator system," in 2009 American Control Conference, 2009, pp. 3488-3493.

[21] E. Tahoumi, F. Plestan, M. Ghanes, and J. Barbot, "New robust control schemes based on both linear and sliding mode approaches: Design and application to an electropneumatic actuator," IEEE Trans. on Control Systems Technology, pp. 1-8, 2019.

[22] C. Qian and W. Lin, "Recursive observer design, homogeneous approximation, and nonsmooth output feedback stabilization of nonlinear systems," IEEE Trans. on Automatic Control, vol. 51, no. 9, pp. 1457$1471,2006$.

[23] C. Qian and W. Lin, "Nonsmooth output feedback stabilization of a class of genuinely nonlinear systems in the plane," IEEE Trans. on Automatic Control, vol. 48, no. 10, pp. 1824-1829, 2003.

[24] B. Yang and W. Lin, "Output feedback stabilization of a class of homogeneous and high-order nonlinear systems," in 42nd IEEE Int Conference on Decision and Control (IEEE Cat. No.03CH37475), vol. 1, 2003, pp. 37-42 Vol.1.

[25] E. Tahoumi, F. Plestan, M. Ganes, and J. P. Barbot, "The implicit discretization of the super-twisting sliding-mode control algorithm," in 2018 European Control Conference, 2018, pp. 2368-2373.

[26] W. Perruquetti and J. P. Barbot, Sliding Mode Control in Engineering (1st ed.). USA: Marcel Dekker, Inc., 2002.

[27] B. Bandyopadhyay, P. S. Gandhi, and S. Kurode, "Sliding mode observer based sliding mode controller for slosh-free motion through pid scheme," IEEE Trans. on Industrial Electronics, vol. 56, no. 9, pp. 3432-3442, 2009.

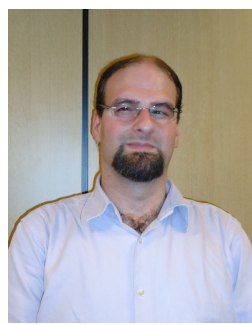

Loïc Michel is currently postdoctoral fellow at the Ecole Centrale de Nantes, France, working on advanced homogeneous control and differentiation techniques. He received his Ph.D. in electrical engineering in 2012 from the University of Quebec, Canada. His research interests include advanced techniques for robust and intelligent control with applications in biology, mechatronic systems and neural networks.

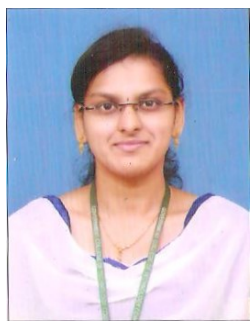

Subiksha Selvarajan received her B.E. degree in Electronics and Insturmentation Engineering in 2018 from Government College of Technology (GCT), Coimbatore, India. She is currently pursuing her Master's degree in Control and Robotics - Control Systems at Ecole Centrale de Nantes (ECN), France. She was working on the application of sliding mode algorithms in the Process Control Laboratory, GCT in 2017- 2018. Since February 2020, she is working on her Master's thesis with the Commande team at LS2N laboratory, ECN. Her research interests include non-linear control and study of observers.

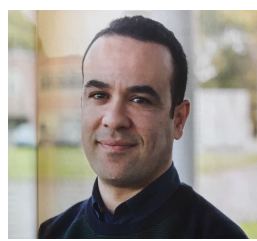

Malek Ghanes received the M.Sc. degree and Ph.D. in applied automatic and informatics both from IRCCyN (now LS2N), Centrale Nantes (CN), in 2002 and 2005, respectively. From September 2005 to September 2006, he was a Postdoctoral position at GReyC. From September 2006 to April 2016 he has been with ECS-Lab, Quartz, ENSEA, France, where he was an Associate Professor and the Head of the Department of Automatic and Electrical Engineering. Since May 2016, he was promoted as a Full Professor at LS2N-ECN. His research interests include observation and control of nonlinear systems, with applications mainly to electric systems. Prof. Ghanes was recipient of the Best Paper Award 2013 from the Journal of Control Engineering Practice (CEP) and the Applied Research Award 2015 from the Federation of Electronic, Electrical and Communications Industries FIEEC. He is currently holding a Chair on Electric Vehicle performances between Renault and CN.

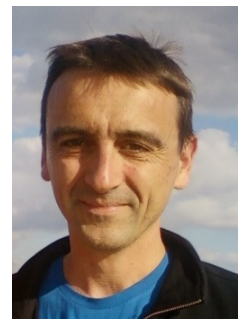

Franck Plestan received the Ph.D. in Automatic Control from the Ecole Centrale de Nantes, France, in 1995. From September 1996 to August 2000, he was with Louis Pasteur University, Strasbourg, France. In September 2000, he joined the Ecole Centrale de Nantes, Nantes, France where he is currently Professor. His research interests include robust nonlinear control and estimation (higher order sliding mode, time delay), and applications (pneumatic actuator, robotics, wind turbine).

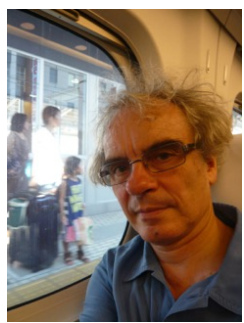

Yannick Aoustin is Full Professor at University of Nantes, France. He earned his Ph.D. degree in Automatic in 1987 and his research degree for leading research and PhD students in 2006. He has been engaged in research for more than 25 years and his research interests include mechanical systems under actuated legged robots, bipedal, nonlinear observers, and biomechanics.

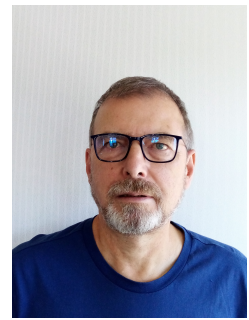

Jean-Pierre Barbot is Professor at ENSEA CergyPontoise, France and he is actually in secondment a LS2N-CNRS Nantes. He was the former director of the Quartz Laboratory EA 7393 and former member of EPI Non-A at INRIA. After his graduation from the Ecole Normale Supérieure of Cachan (France), he received the $\mathrm{Ph}$. D. degree in automatic control from the University of Paris XI (France) in 1989 and the HDR in 1997. These main research activities deal with sliding mode control and observation, system under sampling, hybrid system, delay system, synchronization of chaotic system, normal form and recently sparsity apply to FDI and Cyber-attack detection. These main application domains are electrical machine, power converter and renewable energy. 